\title{
Editorial
}

\section{Gene-environment interactions and public health nutrition}

A non-scientific survey of the scientific literature reveals that the number of articles dealing with gene-environment interactions has exploded over the past decade. A Medline search on the terms 'gene', 'environment' and 'interaction' shows 120 articles published in 1995, 309 in 2000 and 1211 in 2005. In this issue of Public Health Nutrition, two articles join that surge, in some sense marking a shift in the articles we are seeing and publishing in our journal.

Using data from the Swedish Young Male Twins Register, Karnehed et al. ${ }^{1}$ found that low physical activity and low fibre intake were significantly associated with greater waist circumference in their sample. Moreover, they discovered an interaction between genetic susceptibility and physical activity: among twins with higher genetic susceptibility to greater waist circumference, low physical activity appeared to have a greater effect on attained waist circumference than it did among twins at low genetic risk.

In the second of the two articles, Salminen et $a l^{2}$ examined data from a 2- to 3-year family-based health education and counselling intervention. They saw no significant differences in serum lipids between risk (children with $\varepsilon 3 / 4$ or $\varepsilon 4 / 4$ genotype) and non-risk (children with $\varepsilon 2 / 3$ or $\varepsilon 3 / 3$ genotype) groups, with the exception of the total cholesterol/high-density lipoprotein cholesterol ratio among boys. Their findings indicate that the effects of the intervention were similar in children regardless of their apolipoprotein E genotype.

The two articles raise two methodological issues of concern in gene-environment $(G \times E)$ studies: the measurement of genetic factors and the measurement of environmental (or more broadly, non-genetic) factors. Error in the measurement of non-genetic factors is a longstanding problem in epidemiology and is no less a concern in epidemiological studies on $\mathrm{G} \times \mathrm{E}$ interactions. Karnehed et al. discuss the possible implications of error in measuring dietary intake and physical activity in their observational study of male twins. But even in an intervention study, in which we have the greatest control over the 'exposure' of interest, we can raise questions about the exposure that was measured. Would a more intensive intervention have had a more visible effect? Might we have seen different results if the intervention had targeted a more specific age group (e.g. 6-9-year-olds)? In any case, the interpretation of findings for non-genetic factors merits close scrutiny, as it always does.

The measurement of genetic characteristics, likewise, merits close scrutiny. A twin study allows for a clever and efficient analysis, but classifying twins as being at high or low genetic risk based on their concordance in falling above or below the residual median for waist circumference leaves some room for interpretation. To what extent does their concordance reflect similar early-life environment rather than true genetic risk? That the $\mathrm{G} \times \mathrm{E}$ analysis findings for dizygotic twins resembled those for monozygotic twins, in fact, gives some support to the possibility that shared environment contributed to their concordant risk status. Salminen et al. use a different approach to represent the 'gene' in their $\mathrm{G} \times \mathrm{E}$ study by selecting a polymorphism hypothesised a priori to affect cholesterol absorption and plasma lipids. The question that it leaves unanswered, though certainly not by any shortcoming of the study's design, is whether we might expect to find any genes by which responders to such an intervention can be distinguished from non-responders.

A final question that we might ask as readers of Public Health Nutrition is this: are articles on $\mathrm{G} \times \mathrm{E}$ interactions relevant to public health nutrition? A purist might argue that the greatest value of $\mathrm{G} \times \mathrm{E}$ studies is in their potential to elucidate aetiology and to suggest more targeted recommendations and interventions - but public health nutritionists are more concerned with practical implications than with aetiology, with broad population measures rather than with specific, targeted advice.

I would argue that $\mathrm{G} \times \mathrm{E}$ articles are relevant to the field of public health nutrition, and hence to our journal, in three ways. First, the studies generally reaffirm the importance of the environment in $\mathrm{G} \times \mathrm{E}$ studies. Less physical activity, for example, predicted greater waist circumference among male twins regardless of their presumed genetic susceptibility ${ }^{1}$. By itself, confirming the importance of non-genetic factors is of course not reason enough for an article to be published in Public Health Nutrition. But a corollary is that $\mathrm{G} \times \mathrm{E}$ articles in Public Health Nutrition must have a relevant E component and so, in that sense, they retain their broader relevance.

A second reason that $\mathrm{G} \times \mathrm{E}$ articles are relevant is that they provide some sense as to the limits of public health measures. Besides pointing out how unfair genetic inheritance can be - in the study by Karnehed et al., for example, ' 45 minutes of moderate PA may be enough to prevent weight gain in some, whereas 60 minutes might be too little in others' $-\mathrm{G} \times \mathrm{E}$ studies also reveal whether variability in genetic characteristics contributes to variability in the success or failure of public health measures and recommendations. 
Finally, besides the academic reasons generally given for interest in $\mathrm{G} \times \mathrm{E}$ studies - aetiological knowledge, targeted prevention, etc. - there is the desire for knowledge of self. Gene therapy notwithstanding, genes represent the most immutable determinants of ourselves. They set limits on what we are capable of being and doing, and they provide us with our potential in life. $\mathrm{G} \times \mathrm{E}$ studies often and necessarily appear to dwell in the minutiae of biology, in response to which we might wonder if we are being reduced to a single gene or protein. But each study is part of an endeavour to determine the extent to which we are able to maintain our own health and, in the bigger picture, to control our own destinies. Studies at the interface of nutrition and genetics can be worrisome when they appear too far-flung from the realm of public health. Still, such studies inform us about our potential and our limits as public health practitioners (and as ourselves). For that reason they have a place in our journal - a place that will likely grow in coming years.

Marilyn Tseng

Editor

\section{References}

1 Karnehed N, Tynelius P, Heitmann BL, Rasmussen F. Physical activity, diet and gene-environment interactions in relation to body mass index and waist circumference: The Swedish Young Male Twins Study. Public Health Nutrition 2006; 9(7): 851-858.

2 Salminen M, Lehtimäki T, Fan Y-M, Vahlberg T, Kivelä SL. Apolipoprotein E polymorphism and changes in serum lipids during a family-based counselling intervention. Public Health Nutrition 2006; 9(7): 859-865. 